\title{
Prospects of Variable Star Research by Future Space Missions
}

\author{
Laurent Eyer \\ Instituut voor Sterrenkunde, Katholieke Universiteit Leuven, \\ Celestijnenlaan 200 B, B-3001 Leuven, België
}

\begin{abstract}
ESA and NASA are studying projects having a tremendous return on variable star research. Other national space agencies are also studying or developing projects of smaller costs but with impressive returns. The projects range from global Galactic surveys like the ESA mission GAIA which will give photometric time series for about 1 billion stars, to detailed pulsation-mode studies like the CNES mission COROT which could reach a photometric precision lower than $1 \mathrm{ppm}$. The presentation will emphasize the future astrometric, asteroseismological and planet detection missions.
\end{abstract}

\section{Introduction}

Many advantages are present when going in space. The most exploited one has been the access to wavelengths not transmitted through the atmosphere. For photometry near the visible part of the spectrum, there are many benefiting aspects: No atmosphere means no absorption bands of $\mathrm{H}_{2} \mathrm{O}$ and $\mathrm{O}_{3}$, no scintillation, no emission or diffusion from the sky (dark sky), no variable extinction. Furthermore depending on the satellite orbit, it may be possible to make long continuous monitorings (however eclipses by the Moon or the Earth may occur) or to make a global sky coverage.

As a comparative example, the COROT team (see section 6) studied fictive Earth projects (cf. Baglin 1997a) and showed that even an immoderate project of $88 \mathrm{~m}$-class telescopes disseminated around the Earth would not surpass their small space mission.

The drawbacks of space projects are their complexity, cost, aging, limited operation time, and many technical constraints. Space missions are fragile on technical and also political point of views. The global budgets of ESA and NASA in local currencies have been relatively flat for about 8 years, but they may be subjected to high volatility according to changes in the policy.

\section{Main Types of Missions}

Three main types of missions will be presented: one performing astrometry, one oriented towards asteroseismology and one aiming at detecting telluric planets. Many of these projects lead to massive photometric surveys and have thus very 
valuable scientific returns as discussed by Paczyński (1997). Then, an overview of the distinctive features of some specific satellites will be given.

\subsection{Astrometric Missions}

After the pioneering mission Hipparcos, projects performing astrometric measurements are flourishing. Thanks to the advance of technology (CCD, interferometry), the jump in astrometric precision is of 2-3 orders of magnitude, thus making a new mission very attractive. The accuracies reached by these projects are now labeled in $\mu$ as (micro arcsec), cf. Table 1.

The interest for variable stars is threefold. First, several proposed missions are scanning the whole sky and performing photometry. They will thus give, as Hipparcos did, huge amounts of photometric measurements, achieving magnitude completeness. As the scanning law is optimised for the astrometric purpose, the time series have a semiregular sampling which produce aliasing. Second, the information of parallax is a stringent constraint for testing stellar models when used in asteroseismology (cf. Baglin 1997b, Favata 1998). In the case of Hipparcos for instance, Matthews, Kurtz, \& Martinez (1999) showed a comparison of asteroseismological parallax versus trigonometric parallax. On an other hand, absolute luminosities or masses derived from parallaxes can be used as starting point of the seismological models. Third, parallaxes are also of primordial importance for calibrating distance indicators as Cepheids, $R R$ Lyrae and Miras. All the presented missions will literally pin down many of these objects.

Table 1. Summary of several experiments performing astrometry, Mag is the magnitude range or limiting magnitude, $N$ the number of measured stars in million, $\sigma_{\mathrm{A}}$ the astrometric precision in $\mu$ as at $V=9$ and $V=15, T_{\mathrm{D}}$ is the date for realisation decision (Month/Year), $T_{\mathrm{L}}$ is the year of the launch.

\begin{tabular}{lrrrrrr}
\hline Mission & Agency & $\mathrm{Mag}$ & $N$ & $\sigma_{\mathrm{A}}$ & $T_{\mathrm{D}}$ & $T_{\mathrm{L}}$ \\
\hline GAIA & ESA & 20 & 1000 & 4,10 & $\sim 9 / 00$ & 2009 \\
FAME & USNO/NASA & $5-15$ & 40 & 50,500 & $10 / 99$ & 2004 \\
DIVA & Germany & 15 & 35 & 150,5000 & $\sim 12 / 99$ & 2003 \\
\hline
\end{tabular}

\subsection{Asteroseismological Missions}

Several unsuccessful attempts have been made to measure solar-like oscillations from Earth, for such measurements the precision in luminosity determination to be reached is as high as few ppm, furthermore high frequency resolution is required which imposes very long continuous monitoring. As previously remarked, the scintillation is a limiting factor which is magnitude independent as long as photon noise is not dominating. With space missions these requirements can be reached. 


\subsection{Planet Detection Missions}

Among several methods to detect planets, the most famous is the measurement of the periodic variation in radial velocity that a planet induces on its host star. Another promising technique, shown to be more appropriate for the discovery of telluric planets, is the detection of the luminosity decrease during an eclipsing transit of the planet. In this case, the system planet-star should be in a favorable configuration for the observer. Thus large numbers of stars must be observed to achieve valuable statistics.

\section{The GAIA Mission}

The goal of the GAIA satellite is to describe our Galaxy and its history by making an inventory of $1 \%$ of its stars (down to magnitude $V=20$ ) in terms of their kinematical and physical properties. However, the impact of GAIA will go well beyond gaining knowledge of the Galaxy, it will infiltrate many domains of astronomy such as stellar astronomy, general relativity and cosmology. GAIA should not be seen merely as a Super-Hipparcos; it has several instruments among them one is performing astrometry reaching the precision of $4 \mu$ as for magnitude $V=12$ and $10 \mu$ as for magnitude $V=15$. These measurements lead to parallaxes and proper motions. On board, GAIA has a spectrometer which measures radial velocities (with precision of $3-10 \mathrm{~km} / \mathrm{s}$ for $V<17$ ) to complete the 3-dim velocity field. A provisional 11 intermediate band photometric system was specially designed in order to determine the temperature, gravity and metallicity of the observed stars. GAIA will produce photometric time series of about 100 measurements spread over 5 years. The precision is magnitude dependent (cf. Grenon et al. 1999) and for a single transit is of the order of $0.05 \mathrm{mag}$ at $V=17$ in the band F51 (centered at $570 \mathrm{~nm}$ and of $90 \mathrm{~nm}$ width). The time sampling is semi-regular as a result of the scanning law of the satellite. Eyer \& Cuypers (2000) are studying the expectation for variable star numbers. Although the estimations are at preliminary stages, GAIA might detect a few thousand Cepheids and about $90000 \mathrm{RR}$ Lyrae stars. GAIA will permit to study star pulsations in terms of the physical parameters, especially the metallicity. (See http://astro.estec.esa.nl/SA-general/Projects/GAIA/)

\section{FAME}

FAME (http://aa.usno.navy.mil/FAME/) stands for Full-sky Astrometric Mapping Explorer, and it is an accepted NASA MIDEX mission. FAME will observe 40 million stars and will perform a Galactic survey magnitude complete up to $V=15$. The astrometric precision will be $<50 \mu$ as for mag $V<9$ and $<500 \mu$ as for mag $V<15$. It will collect photometric measurements in four bands of the Sloan system (Fukugita et al. 1996). The announced precision of the photometry is $1.6 \mathrm{mmag}$ for $9^{\mathrm{m}}$ and $25 \mathrm{mmag}$ for $15^{\mathrm{m}}$ in the astrometric filter. The scanning law will determine the time sampling. The rotation period of the satellite is 40 minutes, the length of the mission is 2 years and a half. The satellite will furnish thousands of measurement per star, with a semi-regular sampling grouped in sequences of 9-31-9 etc minutes. 


\section{DIVA}

The DIVA (http://www aip.de/groups/DIVA/, Double Interferometer for Visual Astrometry) satellite is a project of the German space agency (DLR). DIVA will reach an astrometric precision of $150 \mu$ as at $V=9$ and 5 mas at $V=15$. DIVA will have broad-band and narrow-band systems. The photometric precision is under study. The scanning law is very similar to the Hipparcos one, with a rotation period of 2 hours.

\section{The COROT Mission}

The COROT (http://www astrsp-mrs.fr/www/pagecorot.html, COnvection and Rotation) satellite has two major goals, first it was designed for doing asteroseismology, focussing on 5 principal fields for very precise and continuous monitoring of 5 months each. The noise level is $0.6 \mathrm{ppm}$ for $\mathrm{G}$ type stars over $5 \mathrm{~d}$. The long time base makes possible the high-accuracy frequency determination $(0.1 \mu \mathrm{Hz})$ necessary for the detailed seismological investigation of stellar structure proposed for COROT. A planet detection program using the transit method was added, this other goal has to survey a large sample of stars $(30000$ to 60000 ) with a photometric precision of $0.1 \%$ for $16 \mathrm{~min}$ of integration time in order to detect telluric planets. This survey is achieved in dense fields down to $15^{\mathrm{m}}$. Two-color information will be available for $\sim 1 / 3$ of the targets to establish the achromaticity of the phenomenon. There is also an exploratory program where COROT will measure 50 to 80 solar-like stars with $V<9$ at a precision better than $2.4 \mathrm{ppm}$.

\section{MONS}

MONS (http://www.obs.aau.dk/MONS/, Measuring Oscillation in Nearby Stars) is a project of the Danish Small Satellite Program, which already launched one successful satellite (Ørsted). MONS will observe about 20 solar-like stars over two years as a primary scientific objective; it will also measure $\delta$ Scuti, roAp stars and samples of stars of all types as a secondary objective. Its eccentric orbit with high apogee (Molniya orbit) permits access to a large part of the sky. It will measure changes in colour ratio. Oscillations of amplitude of 1-10 ppm will be detected (cf. Kjeldsen, Bedding, \& Christensen-Dalsgaard 1999). Furthermore, MONS will obtain science data from the Star Imagers designed for attitude control; they will permit measurement of about 900 stars, 700 with the Imager SI 1, and 200 with the Imager SI 2. The accuracy will be about 11 ppm (SI 1) and $26 \mathrm{ppm}$ (SI 2) at magnitude $V=6$.

\section{MOST}

MOST (http://ww .astro.ubc.ca/MOST/, Microvariability and Oscillations of STars) is a funded project which is the first space telescope of the Canadian Space Agency. MOST will observe several (3-5) solar-like stars over 40 days. It will also observe roAp, Wolf-Rayet and $\delta$ Scuti stars. The photometric precision for a $V=6$ star is better than a few ppm for 10 days of integration. 


\section{Kepler}

Although the Kepler project (http://www .kepler.arc.nasa.gov/) was not selected due to questions about the ability to perform the photometry in space, the scientific case was rated very highly. The Kepler team is planning to resubmit the project. Its goal is to "explore the structure and diversity of planetary systems" by performing differential photometry. It will monitor 100000 mainsequence stars in the magnitude range from 9th to 14th with an accuracy of $0.002 \%$ including instrument noise, shot noise and stellar variability for a star of $V=12$. The mission would point continuously at a single field in Cygnus during 4 years. Because only data for preselected stars are saved, all objects to be monitored must be pre-specified. The team will probably entertain "guest observing" for additional interesting objects like variable stars. A subset of a few hundred brighter stars will also be incorporated and observed with a shorter integration time for asteroseismological purposes.

\section{Conclusion}

Many of these projects might be selected in a near future for their realisation. The projects performing astrometry will provide deep galactic surveys and thus an inventory for galactic variable stars. The asteroseismological projects have common goals but they use different ingenious techniques and have different and diverse by-products.

The future spatial projects will open new domains in variable star studies, therefore precise predictions are delicate. Since some presented missions are already funded, there is the conviction that after the projects like Hipparcos or the microlensing surveys, the domain of variable stars will still be under a strong evolution.

\section{References}

Baglin, A. 1997a, CNES, COR-SP-0-83-PROJ

Baglin, A. 1997b, in Highlights of Astronomy, Vol. 11A, ed. J. Andersen, in The First Results of Hipparcos and Tycho (JD 14 of the XXIII General Assembly of the IAU), ed. C. Turon (Dordrecht: Kluwer), 555

Eyer, L. \& Cuypers, J. 2000, in these proceedings, p. 71

Favata, F. 1998, in The First MONS Workshop: Science with a Small Space Telescope, ed. H. Kjeldsen \& T. R. Bedding (Aarhus: Aarhus Universitet), 89

Fukugita, M., Hichikawa, T., Gunn, J. E., et al. 1996, AJ, 111, 1748

Grenon, M., Jordi, C., Figueras, F., \& Torra, J. 1999, to be published

Kjeldsen, H., Bedding, T. R., \& Christensen-Dalsgaard, J. 1999, Document No. MONS-99/01

Matthews, J. M., Kurtz, D., \& Martinez, P. 1999, ApJ, 511, 422

Paczyński, B. 1997, in Variables Stars and the Astrophysical Returns of the Microlensing Surveys (12th IAP Astrophysics Colloquium), ed. R. Ferlet, J.-P. Maillard, \& B. Raban (Gif sur Yvette: Editions Frontières), 357 